\title{
Current Status on Cholangiocarcinoma and Gallbladder Cancer
}

\author{
Tomoki Ebata ${ }^{a}$ Giorgio Ercolani ${ }^{b}$ Domenico Alvaroc \\ Dario Ribero $^{d} \quad$ Luca Di Tommaso $^{\text {e Juan W. Valle }}$
}

aDivision of Surgical Oncology, Department of Surgery, Nagoya University Graduate School of Medicine, Nagoya, Japan, 'bepartment of Medical and Surgical Sciences, Hospital Sant'orsola-Malpighi, University of Bologna, Bologna, 'Division of Gastroenterology, Department of Medico-Surgical Sciences and Biotechnologies, University Sapienza of Rome, Rome, dDepartment of Hepatobiliary and Pancreatic Surgery, European Institute of Oncology, Milan, ePathology Unit, Humanitas Clinical and Research Center, Milan, Italy, Institute of Cancer Sciences, University of Manchester and Department of Medical Oncology, The Christie, Manchester, United Kingdom

\section{Key Words}

Biliary tract cancer $\cdot$ Cholangiocarcinoma $\cdot$ Gallbladder cancer

\begin{abstract}
Background: Cholangiocarcinomas (CC) as well as gallbladder cancers are relatively rare and intractable diseases. Clinical, pathological, and epidemiological studies on these tumors have been under investigation. The current status and/or topics on biliary tract cancers have been reported in the East West Association of Liver Tumor (EWALT), held in Milano, Italy in 2015. Summary: All the authors, herein, specifically reported the current status and leading-edge findings on biliary tract cancers as the following sequence: epidemiology of $\mathrm{CC}$, surgical therapy for intrahepatic CC, surgical therapy for perihilar CC, surgical therapy for gallbladder cancer, chemotherapy for biliary tract cancers, and new histological features in CC. Key Message: The present review article will update the knowledge on biliary tract cancers, enhancing the quality of daily clinical practice. However, many features about these cancers remain unknown; further studies are required to establish disease-specific optimal treatment strategies.

Copyright $\odot 2016$ S. Karger AG, Basel
\end{abstract}




\section{Epidemiology of Cholangiocarcinoma (CC)}

The epidemiology of CC and its subtypes displays enormous geographic differences reflecting the distribution of different risk factors, both environmental and genetic alike [1-3]. Thus, CC is exceptionally common in Chile, Bolivia, South Korea and North Thailand while, it is a rare cancer (incidence less than 6 cases per 100,000) in Western countries. The global incidence of CC varies, from rates of 113 cases per 100,000 in Thailand to 0.1 cases per 100, 000 in Australia [4]. CC generally occurs after the fourth decade of life, and aging is directly associated with a progressive increase in the incidence, with the risk being slightly higher in men than women $[1,2]$. The epidemiologic profile of CC subtypes, namely intrahepatic CC (ICC) and extrahepatic CC (ECC), is largely biased by the misclassification of perihilarCC as ICC rather than ECC $[2,3]$, potentially leading to an overestimation of the incidence of ICC and an underestimation of ECC. A progressive increase of the incidence of ICC was reported until the end of the last century, with it then plateauing in the last decade $[1-3,5]$. The rising incidence has been linked with the burden of hepatitis $\mathrm{C}$ virus (HCV) infection [1]. In contrast, the incidence of ECC has tended to progressively decrease. Interestingly, in the last several years, the mortality from this cancer has tended to be lower than the incidence (especially for ICC) suggesting better clinical management. As risk factors for CC, HCV infection, hepatitis B virus infection, and hepatolithiasis are specifically associated with ICC [1-3, 5-7]; an abnormal pancreaticobiliary junction and choledochal cysts are associated with ECC, while liver flukes and primary sclerosing cholangitis have been associated with both malignancies $[2,5]$. In addition, an 'outbreak' of CC occurred among young adult workers in the offset color proof-printing department at a printing company in Japan. In this respect, two carcinogenic chemicals (1,2-dichloropropane and/or dichloromethane) were found to cause CC in humans [8]. However, in most patients with CC no putative risk factor(s) can be detected at the diagnosis.

\section{Surgical Treatment}

As there has been little progress in the development of efficacious chemotherapy and locoregional treatments, patients with $\mathrm{CC}$ or gallbladder cancer should be offered surgical resection that represents the only treatment associated with long-term survival and the possibility for cure. Achieving a tumor-free surgical margin, which forms one of the robust prognostic indicators, is a primary goal of resection for these tumors. Resectability criteria have remained poorly defined, and vary among institutions and countries. However, localized tumors or less are generally considered as resectable whereas locally advanced tumors are not. Distant metastatic or stage IVB diseases are far-advanced disease beyond the scope of surgery, whereas Nodal metastatic disease remains controversial. As patient-related factors, good performance status of 0 or 1, low American Society of Anesthesiologists Physical Status, and satisfactory functional reserve of the future liver remnant are required for safety. Disease-specific issues are discussed in the each section.

\section{Surgical Treatment for ICC}

Surgical indication for ICC with multiple intrahepatic tumors remains controversial [9]. Several case series have shown that the presence of multiple tumors, found in up to $44 \%$ of the patients, is one of the strongest negative prognostic factors. However, different diseases are grouped into the category "multiple tumors" including satellite nodules, synchronous multicentric tumors and intrahepatic metastases, which theoretically have different natural 
histories. In the absence of robust evidence, only patients with satellite nodules should be considered for resection. The implication of this technically-based definition of resectability is to classify a number of patients who have advanced, complex tumors as potential candidates for surgery [10]. With an aggressive surgical approach, that might require extensive hepatectomies and in some cases major vascular and biliary resections, high rates of microscopically margin-negative for tumor (R0) resections have been reported [9-11]. The reported 5-year survival rates are $67 \%$ for T1 (solitary tumor without vascular invasion) tumors, $38 \%$ for T2 (solitary tumor with vascular invasion or multiple tumors) tumors, 35\% for T3 (tumor perforating the visceral peritoneum or involving the local extrahepatic structures) tumors, and $41 \%$ for T4 (tumor with periductal invasion) tumors [12], respectively. Recent data also suggest a survival benefit for patients undergoing repeat resection for liver recurrences. In the absence of studies defining the best treatment approach (systemic, regional or local including surgery and thermal ablation) a repeat hepatectomy might be considered in highly selected patients [13]. Although several clinicopathologic features have been demonstrated to affect long-term survival, one of the major determinants of poor prognosis is the presence of lymph node metastases. In cases of locoregional nodal involvement, which is as high as 39\% of patients $[10,13]$, liver resection should still be considered albeit the evidence of grossly positive lymph nodes on preoperative imaging might suggest approaching these patients with caution, considering chemotherapy first. Because of the prognostic impact of nodal metastases, a routine assessment of the hepatoduodenal lymph node is suggested by the majority of experts $[10,13]$. Nevertheless, the extent of nodal retrieval, from sampling only radiographic or clinically suspicious nodes to a formal lymphadenectomy, is still controversial. Despite the lack of evidence of a survival advantage associated with a systematic lymphadenectomy, Eastern authors and most of the Western ones do perform at least a complete dissection of the hepatoduodenal ligament to accurately stage the $\mathrm{N}$ parameter, which in turn may assist in the decision for adjuvant therapies.

\section{Surgical Treatment for Perihilar CC}

Hilar bile duct resections with or without a limited hepatectomy have been performed previously. However, this procedure was likely to result in positive margins, yielding unsatisfactory long-term survival rates. Recent advances in surgical techniques, presurgical management, and the understanding of tumor pathology have driven hepatobiliary surgeons to perform more extended procedures involving hemihepatectomy or more extended hepatectomy, caudate lobectomy, extrahepatic bile duct resection, and regional lymphadenectomy [14].

The type of hepatectomy performed has been organized into four typical procedures namely: 1) left hepatectomy, 2) left trisectionectomy, 3) right hepatectomy, and 4) right trisectionectomy, which are selected based on the lateral tumor extension representing the Bismuth classification. Hemihepatectomies are indicated for Bismuth type I to III tumors whereas hepatic trisectionectomies are indicated for Bismuth type IV tumors. Although in the past Bismuth type IV tumors have been considered as unresectabe disease, this concept is no longer a valid one. In leading centers, resection and reconstruction of the portal vein and hepatic artery, and combined pancreaticoduodenectomy have been attempted to eradicate advanced tumors $[15,16]$. However, the indication varies among institutions, suggesting that these procedures are yet to be standardized.

The overall survival rate after surgery exclusively depends on the tumor stage. The 5-year survival rates are $100 \%$ for stage $0,87 \%$ for stage I, $61 \%$ for stage II, $45 \%$ for stage IIIA, 30\% for stage IIIB, 35\% for stage IVA, and 11\% for stage IVB [17], respectively. Nodal metastasis is one of the robust prognostic factors, and the incidence of node-positivity reaches approximately $50 \%$. The 5 -year survival rate for node-positive patients is around $20 \%$ whereas in contrast, that for node-negative patients is approximately $50 \%$ [18]. Therefore, postoperative 
chemotherapy is often given to patients with nodal metastases, although level-1 evidence supporting this practice is lacking at present.

\section{Surgical Treatment for Gallbladder Cancer}

Gallbladder cancer is the most frequent tumor originating from the extrahepatic biliary tract. Most gallbladder cancers are adenocarcinomas (80\%). The tumor tends to invade the adjacent structures including the liver parenchyma, bile duct, and major vessels; perineural tissue and regional lymph node are often invaded. The extension of the tumor is defined according to the American Joint Committee on Cancer classification (7th edition) [19] based on ultrasonography, computed tomography scanning and/or magnetic resonance imaging. There are few studies evaluating the role of positron emission tomography scanning in patients with gallbladder cancer. Staging laparoscopy may be useful to avoid unnecessary laparotomy [20].

Surgical procedures depend on tumor stages. T1a (tumor invading lamina propria) tumors are radically treated by simple cholecystectomy; T1b (tumor invading muscle layer) tumors are treated by gallbladder bed resection and regional lymphadenectomy. In T2 (tumor invading perimusclar connective tissue) or more advanced tumors, minimal hepatectomy is performed to achieve R0 resection, because the extent of liver resection does not correlate with long term survival. Mostly, resection of segments 4 a and 5 is sufficient; yet, right hepatectomy may be required in patients with extensive liver invasion or vascular invasion (right portal vein or hepatic artery). Whenever jaundice is present, or the stump of the cystic duct is positive for tumor invasion, bile duct resection followed by hepaticojejunostomy is mandatory [21], or otherwise, it should not be recommended. Regional lymphadenectomy is usually performed around the hepatic pedicle along the common hepatic artery and in the retropancreatic space. A minimum of six lymph nodes should be harvested to obtain an adequate staging of the disease. Finally, one of the most extended procedures, hepatopancreaticoduodenectomy, should be undertaken only in highly selected patients, because the morbidity and mortality rates are very high and a survival benefit has not been shown [22]. Finally, the approximate survival rates at 5 years after resection are shown according to tumor stages: $90 \%$ for T1 tumors, $60 \%$ for T2 tumors, 20 to $25 \%$ for T3 (tumor penetorating the serosa or invading the one adjacent organ) tumors, and $<10 \%$ for T4 (tumor invading main portal vein, hepatic artery, or two or more adjacent organs) tumors [23], respectively.

\section{Chemotherapy}

CC and gallbladder cancer were, for many years, considered to be unresponsive to chemotherapy largely due to the lack of quality data supporting its use. Most patients present with advanced, inoperable disease and without treatment the median survival is about three months. Two studies have shown chemotherapy to confer a survival advantage over best supportive care. A pooled analysis of historical studies identified gemcitabine, fluoropyrimidines and platinum agents as beneficial agents [24]. Combination chemotherapy with cisplatin and gemcitabine (CisGem) has been shown to improve overall survival over gemcitabine monotherapy in a phase III (ABC-02) study [25], with benefit seen across all exploratory subgroups in this study, as well as in a Japanese randomized study [26] using the same regimen. Unfortunately, there are no predictive biomarkers for selection of patients most likely to benefit from chemotherapy. Patients with obstructive jaundice (bilirubin $>1.5 \mathrm{x}$ upper limit of normal) were excluded from the ABC-02 study; recent data have shown that CisGem may benefit performance status $0-1$ patients with elevated bilirubin levels due to malignant 
obstructive endoluminal disease [27] versus liver parenchymal replacement by tumor, which shows no benefit.

The survival advantage with first-line chemotherapy is modest (approx. 1 year) and further treatment options are required. At present, there is no established second-line chemotherapy. A recent systematic review of second-line chemotherapy showed that $<10 \%$ of patients achieved an objective response and the median progression-free survival was only 3.2 months, coinciding with the first reassessment scan in most patients [28]. Ongoing studies will establish the role of second line chemotherapy, as well as targeted therapies aimed at specific molecular subgroups of patients.

In selected patients with initially unresectable disease, salvage surgery may be possible in the event of a radiological response to neoadjuvant chemotherapy. However this may be a more frequent occurrence with the development of regimens with greater response rates than are seen with CisGem (26\%).

There is no established role for adjuvant chemotherapy although practice varies widely with adjuvant chemotherapy sometimes being considered for patients with lymph nodepositive or margin-positive disease. The results of two completed studies (with either capecitabine [NCT00363584] or gemcitabine and oxaliplatin [NCT01313377] and two ongoing studies with gemcitabine [UMIN 000000820] or with tegafur gimeracil-oteracil potassium [UMIN000011688] may change future standards of care, if positive.

\section{New Histological Features in CC}

CC arises from the epithelium lining bile ducts and peribiliary glands. Conventional ICC is an invasive adenocarcinoma composed of neoplastic cells resembling biliary epithelium arranged in tubules, acini and micropapillae in a variable amount of desmoplastic and inflammatory background. ICC can be further classified as small and large bile duct types [29]. In the former, small-sized tubules and acini invade the liver parenchyma. In the latter, the disease mainly involves the intrahepatic large bile ducts. The neoplastic cells spread along their lumen with flat or micropapillary architecture, infiltrate the duct wall in variable sized tubules or acini, and less frequently invade the surrounding liver parenchyma where it resembles the small bile duct type. Another histological variation of ICC is the bile ductular type, in which neoplastic glands strongly resembles reactive ductules. The small neoplastic cells are arranged in cord-like structures with a slit-like lumen and arborizations, replacing the hepatocytes. Ductal plate malformation type is another variant of ICC with minimal deviation features, reminiscent of findings observed in congenital hepatic fibrosis or Caroli disease [30].

Most perihilar and distal CCs do not differ from conventional ICC. However, they are classified separately because of peculiar aspects $[19,31]$. In the papillary type, the lesion grows intraluminally. Histologically, the tumor is composed of papillary excrescence of neoplastic cells (in situ component, see precursors lesions) and, sometimes, infiltrating foci with ordinary tubular pattern and, not infrequently, a mucinous component. Also the tubular type is characterized by an exophytic lesion which presents with tubules rather than papillae, and the infiltrating component has identical features of classical CC. Finally in the superficial spreading type, neoplastic cells spread on the luminal surface of the bile ducts with only occasional infiltration of the wall, a finding which explains the inconspicuous gross findings of these lesions. Rare histological variants, squamous and sarcomatous differentiation as well as histologic grade 3 are related to a poor prognosis, while patients with mucinous carcinoma have a better prognosis. 
Four types of precursors lesion of CC are reported: 1) flat (biliary intraductal neoplasia [Bil-IN]), 2) papillary (intraductal papillary neoplasm of the bile duct [IPNB]), 3) tubular (intraductal tubular neoplasm of the bile [ITNB]), and 4) mucinous cystic (hepato-biliary mucinous cystic neoplasia [hbMCN]) types; however, these are strongly related to perihilar and distal CCs [30]. Bil-IN is histologically characterized by the presence of epithelial cells with multilayering of nuclei and micropapillary projections. IPNB is characterized by the presence of papillary or villous excrescences, including biliary papilloma and papillomatosis. The lining epithelium may exhibit intestinal, gastric, pancreaticobiliary and oncocytic features. ITNB is a lesion closely related to IPNB, showing a tubular rather than papillary growth, which has been recently suggested as a possible precursor of the former aforementioned disease. Finally, hbMCN is a cyst-forming tumor, usually without communication with bile ducts, with an ovarian-like subepithelial stroma, lined by mucinous cells. All these precursor lesions may show different degrees of cytological atypia (increased nuclear to cytoplasmic ratio, loss of nuclear polarity, nuclear hyperchromasia) turning into a 3-grade system classification distinguishing low- intermediate- and high-grade lesions. When an invasive carcinoma is associated with these precursor lesions, it should be separately designated.

\section{Summary}

Biliary tract cancers are a heterogeneous group of neoplasms that arise in diverse levels in the biliary tree, develop through carcinogenesis, and exhibit variable histologic morphologies and immunohistochemical profiles. A standardized or common therapeutic approach for this devastating disease is yet to be established. Disease-specific optimal treatment will be personalized or diversified according to the patient or tumor status in the future. As anticipated from this article, unfortunately, we do not know much about biliary tract cancers at the present time. Further studies will be required to determine the best modalities for treatment in the future.

\section{Conflict of Interest}

The authors declare that they have no conflict of interest and sponsorship.

\section{References}

1 Bergquist A, von Seth E: Epidemiology of cholangiocarcinoma. Best Pract Res Clin Gastroenterol 2015;29:221-232.

2 Cardinale V, Semeraro R, Torrice A, Gatto M, Napoli C, Bragazzi MC, Gentile R, Alvaro D: Intra-hepatic and extra-hepatic cholangiocarcinoma: New insight into epidemiology and risk factors. World J Gastrointest Oncol 2010;2:407-416.

3 Alvaro D, Crocetti E, Ferretti S, Bragazzi MC, Capocaccia R, AISF Cholangiocarcinoma committee: Descriptive epidemiology of cholangiocarcinoma in Italy. Dig Liver Dis 2010; 42: 490-495.

4 Rizvi S, Gores GJ: Pathogenesis, diagnosis, and management of cholangiocarcinoma. Gastroenterology 2013;145:1215-1229.

5 Tyson GL, El-Serag HB: Risk factors for cholangiocarcinoma. Hepatology 2011;54:173-184.

6 Kobayashi M, Ikeda K, Saitoh S, Suzuki F, Tsubota A, Suzuki Y, Arase Y, Murashima N, Chayama K, Kumada $\mathrm{H}$ : Incidence of primary cholangiocellular carcinoma of the liver in Japanese patients with hepatitis $\mathrm{C}$ virus-related cirrhosis. Cancer 2000;88:2471-2477.

7 Tanaka M, Tanaka H, Tsukuma H, Ioka A, Oshima A, Nakahara T: Risk factors for intrahepatic cholangiocarcinoma: a possible role of hepatitis B virus. J Viral Hepat 2010;17:742-748. 
8 Kumagai S, Kurumatani N, Arimoto A, Ichihara G: Cholangiocarcinoma among offset colour proof-printing workers exposed to 1,2-dichloropropane and/or dichloromethane. Occup Environ Med 2013;70:508-510.

9 Weber SM, Ribero D, O’Reilly EM, Kokudo N, Miyazaki M, Pawlik TM: Intrahepatic cholangiocarcinoma: expert consensus statement. HPB (Oxford) 2015;17:669-680.

10 Ribero D, Pinna AD, Guglielmi A, Ponti A, Nuzzo G, Giulini SM, Aldrighetti L, Calise F, Gerunda GE, Tomatis M, Amisano M, Berloco P, Torzilli G, Capussotti L, Italian Intrahepatic Cholangiocarcinoma Study Group: Surgical Approach for Long-term Survival of Patients With Intrahepatic Cholangiocarcinoma: A Multi-institutional Analysis of 434 Patients. Arch Surg 2012;147:1107-1113.

11 Farges O, Fuks D, Boleslawski E, Le Treut YP, Castaing D, Laurent A, Ducerf C, Rivoire M, Bachellier P, Chiche L, Nuzzo G, Regimbeau JM: Influence of surgical margins on outcome in patients with intrahepatic cholangiocarcinoma: a multicenter study by the AFC-IHCC-2009 study group. Ann Surg 2011;254:824-829, discussion 830

12 Uenishi T, Ariizumi S, Aoki T, Ebata T, Ohtsuka M, Tanaka E, Yoshida H, Imura S, Ueno M, Kokudo N, Nagino M, Hirano S, Kubo S, Unno M, Shimada M, Yamaue H, Yamamoto M, Miyazaki M, Takada T: Proposal of a new staging system for mass-forming intrahepatic cholangiocarcinoma: a multicenter analysis by the Study Group for Hepatic Surgery of the Japanese Society of Hepato-Biliary-Pancreatic Surgery. J Hepatobiliary Pancreat Sci 2014;21:499-508.

13 de Jong MC, Nathan H, Sotiropoulos GC, Paul A, Alexandrescu S, Marques H, Pulitano C, Barroso E, Clary BM, Aldrighetti L, Ferrone CR, Zhu AX, Bauer TW, Walters DM, Gamblin TC, Nguyen KT, Turley R, Popescu I, Hubert C, Meyer S, Schulick RD, Choti MA, Gigot JF, Mentha G, Pawlik TM: Intrahepatic cholangiocarcinoma: an international multi-institutional analysis of prognostic factors and lymph node assessment. J Clin Oncol 2011;29:3140-3145.

14 Ito T, Ebata T, Yokoyama Y, Igami T, Sugawara G, Mizuno T, Shimoyama Y, Nagino M: The pathologic correlation between liver and portal vein invasion in perihilar cholangiocarcinoma: evaluating the oncologic rationale for the American Joint Committee on Cancer definitions of T2 and T3 tumors. World J Surg 2014;38:3215-3221.

15 Ebata T, Yokoyama Y, Igami T, Sugawara G, Takahashi Y, Nimura Y, Nagino M: Hepatopancreatoduodenectomy for cholangiocarcinoma: a single-center review of 85 consecutive patients. Ann Surg 2012;256:297-305.

16 Nagino M, Nimura Y, Nishio H, Ebata T, Igami T, Matsushita M, Nishikimi N, Kamei Y: Hepatectomy with simultaneous resection of the portal vein and hepatic artery for advanced perihilar cholangiocarcinoma: an audit of 50 consecutive cases. Ann Surg 2010;252:115-123.

17 Ebata T, Kosuge T, Hirano S, Unno M, Yamamoto M, Miyazaki M, Kokudo N, Miyagawa S, Takada T, Nagino M: Proposal to modify the International Union Against Cancer staging system for perihilar cholangiocarcinomas. Br J Surg 2014;101:79-88.

18 Nagino M, Ebata T, Yokoyama Y, Igami T, Sugawara G, Takahashi Y, Nimura Y: Evolution of surgical treatment for perihilar cholangiocarcinoma: a single-center 34-year review of 574 consecutive resections. Ann Surg 2013;258:129-140.

19 Edge SB, Byrd DR, Compton CC, Fritz AG, Greene FL, Trotti A, editors: AJCC Cancer Staging Manual. 7th ed. New York: Springer; 2010. 201-225

20 Agarwal AK, Kalayarasan R, Javed A, Gupta N, Nag HH: The role of staging laparoscopy in primary gall bladder cancer-an analysis of 409 patients: a prospective study to evaluate the role of staging laparoscopy in the management of gallbladder cancer. Ann Surg 2013;258:318-323.

21 Nishio H, Ebata T, Yokoyama Y, Igami T, Sugawara G, Nagino M: Gallbladder cancer involving the extrahepatic bile duct is worthy of resection. Ann Surg 2011;253:953-960.

22 Ebata T, Nagino M, Nishio H, Arai T, Nimura Y: Right hepatopancreatoduodenectomy: improvements over 23 years to attain acceptability. J Hepatobiliary Pancreat Surg 2007;14:131-135.

23 Ishihara S, Horiguchi A, Miyakawa S, Endo I, Miyazaki M, Takada T: Biliary tract cancer registry in Japan from 2008 to 2013. J Hepatobiliary Pancreat Sci 2015. doi: 10.1002/jhbp.314

24 Eckel F, Schmid RM: Chemotherapy in advanced biliary tract carcinoma: a pooled analysis of clinical trials. Br J Cancer 2007;96:896-902.

25 Valle J, Wasan H, Palmer DH, Cunningham D, Anthoney A, Maraveyas A, Madhusudan S, Iveson T, Hughes S, Pereira SP, Roughton M, Bridgewater J, ABC-02 Trial Investigators: Cisplatin plus gemcitabine versus gemcitabine for biliary tract cancer. N Engl J Med 2010;362:1273-1281.

26 Okusaka T, Nakachi K, Fukutomi A, Mizuno N, Ohkawa S, Funakoshi A, Nagino M, Kondo S, Nagaoka S, Funai J, Koshiji M, Nambu Y, Furuse J, Miyazaki M, Nimura Y: Gemcitabine alone or in combination with cisplatin in patients with biliary tract cancer: a comparative multicentre study in Japan. Br J Cancer 2010;103:469-474.

27 Lamarca A, Benafif S, Ross P, Bridgewater J, Valle JW: Cisplatin and gemcitabine in patients with advanced biliary tract cancer $(\mathrm{ABC})$ and persistent jaundice despite optimal stenting: Effective intervention in patients with luminal disease. Eur J Cancer 2015;51:1694-1703.

28 Lamarca A, Hubner RA, David Ryder W, Valle JW: Second-line chemotherapy in advanced biliary cancer: a systematic review. Ann Oncol 2014;25:2328-2338.

29 Komuta M, Govaere O, Vandecaveye V, Akiba J, Van Steenbergen W, Verslype C, Laleman W, Pirenne J, Aerts R, Yano H, Nevens F, Topal B, Roskams T: Histological diversity in cholangiocellular carcinoma reflects the different cholangiocyte phenotypes. Hepatology 2012;55:1876-1888.

30 Nakanuma Y, Sato Y, Harada K, Sasaki M, Xu J, Ikeda H: Pathological classification of intrahepatic cholangiocarcinoma based on a new concept. World J Hepatol 2010;2:419-427.

31 Bosman FT, Carneiro F, Hruban RH, Theise ND, editors: WHO Classification of Tumours of the Digestive System 4th ed. Lyon: International Agency for Reseach on Cancer; 2010. 